PROCEEDINGS OF THE

AMERICAN MATHEMATICAL SOCIETY

Volume 132, Number 8, Pages 2233-2240

S 0002-9939(04)07421-0

Article electronically published on March 25, 2004

\title{
ON THE DISCRETE GROUPS OF MOONSHINE
}

\author{
JOHN CONWAY, JOHN MCKAY, AND ABDELLAH SEBBAR
}

(Communicated by Wen-Ching Winnie Li)

\begin{abstract}
We characterize the 171 discrete subgroups of $\mathrm{PSL}_{2}(\mathbb{R})$ occurring in Monstrous Moonshine in terms of their group-theoretic properties alone.
\end{abstract}

\section{THE MAIN RESULT}

Let $\mathbb{M}$ denote the largest sporadic simple group known as the Monster. It was predicted to exist by Fischer and Griess in the early 1970s and was constructed by Griess a few years later 14]. In their Monstrous Moonshine paper [7, Conway and Norton expand observations of McKay and Thompson to formulate the Moonshine conjecture:

(1) To each cyclic group $\langle m\rangle, m \in \mathbb{M}$, is associated a function:

$$
f_{m}(\tau)=\frac{1}{q}+a_{1} q+a_{2} q^{2}+\cdots, a_{k} \in \mathbb{Z}, q=e^{2 \pi i \tau}, \Im \tau>0,
$$

such that each $q$-coefficient is the value of a character (known as the head character) of $\mathbb{M}$ at $m$.

(2) Each $f_{m}$ is a principal modulus for a certain genus zero congruence group commensurable with the modular group, $\Gamma=\mathrm{PSL}_{2}(\mathbb{Z})$.

In fact, for each $\langle m\rangle$, Conway and Norton proposed two discrete subgroups of $\mathrm{PSL}_{2}(\mathbb{R})$ attached to $f_{m}$ defined as follows.

The first group, $F_{m}$, is the invariance group of $f_{m}$. Let $N$ be the level of $F_{m}$, that is, the least $N$ for which $\Gamma(N) \subset F(m)$. Write $h=N / n$ where $n$ is the order of $m$. It happens that $h$ is always an integer such that $h \mid 24$ and $h^{2} \mid N$. The second group, $E_{m}$, is the subgroup of $\mathrm{PSL}_{2}(\mathbb{R})$ whose elements multiply $f_{m}$ by $h$ th roots of unity. Hence $F_{m}$ is a subgroup of $E_{m}$. There are 171 distinct $f_{m}$ of which only 48 occur with $h>1$. In [7], it is proposed that the $E_{m}$ coincide with congruence subgroups $\Gamma_{0}(n \mid h)+\cdots$, and $F_{m}$ is a subgroup of index $h$ in $E_{m}$ with a similar parametrization of the form $\Gamma_{0}(n \| h)+\cdots$ (see Section 2 for the definitions). When $h=1$, we have $E_{m}=F_{m}$ and it is easy to exhibit a principal modulus for the corresponding parametrization (referred to as fundamental); these principal moduli are given by the $\eta$-products and theta functions of Table 3 in 7]. The fundamental elements can be modified to provide principal moduli for the parametrizations of $F_{m}$ even when $h \neq 1$; see Section 6 of [7]. To verify that

Received by the editors August 2, 2002 and, in revised form, May 7, 2003.

2000 Mathematics Subject Classification. Primary 11F22, 11F03; Secondary 30F35, 20 C34.

Key words and phrases. Monster, Moonshine, discrete groups, principal moduli. 
these parametrizations do coincide with the proposed groups, it is enough to check that the first few coefficients of their principal moduli coincide with the first few coefficients of the $f_{m}$. This follows from the automorphic properties of the principal moduli and the replication properties of the $f_{m}$ established by Borcherds together with Norton's theorem that 12 of the first 23 coefficients of a replicable function determine the function completely.

Also, for each group $\Gamma_{0}(n \| h)+\cdots$, Norton has computed a set of generating matrices which fix the corresponding $f_{m}$ for a wider class of functions (the rational replicable functions).

In the Monstrous Moonshine paper, [7], the correspondence was incomplete between the conjugacy classes of cyclic subgroups of the Monster and the genus zero groups $\Gamma_{0}(n \| h)+\cdots$. For $h=1$ it was complete except for three groups referred to as the ghosts. We now provide a simple new criterion on the groups which makes the correspondence exact when $h>1$, and another one which exorcises the ghosts; so the correspondence is now a perfect bijection. In other words, we exhibit simple necessary and sufficient conditions for a discrete subgroup of $\mathrm{PSL}_{2}(\mathbb{R})$ to be the invariance group of a Monstrous Moonshine function.

The Moonshine conjectures have been proved by Borcherds using vertex algebras [3], and this led to the development of several new areas in mathematics as well as applications and connections with physics. Examples of these connections are the vertex algebras and their automorphism groups, hyperbolic Lie algebras, automorphic forms and infinite products, orbifold conformal field theory, generalized Moonshine, elliptic genera, and mirror symmetry. For more on these connections see [3, [4, [9], 8], 11], 15], 16], 17], 20], and [21. All of these connections illustrate the rich nature of the Monster and Moonshine; and while some of these help in understanding Moonshine, many of the connections with other areas are still conjectural or poorly understood. That is to say, the real nature of Moonshine is still remote. Here we provide the answer to one question: What are these modular functions $f_{m}$ (or equivalently their invariance groups since they are principal moduli) attached to elements of the Monster?

Theorem. A modular function occurs in Moonshine if and only if its invariance group

(1) is genus zero,

(2) has the form $\Gamma_{0}(n \| h)+e, f, g, \ldots$,

(3) its quotient by $\Gamma_{0}(n h)$ is a group of exponent 2 , and

(4) each cusp can be mapped to $\infty$ by an element of $P S L_{2}(\mathbb{R})$ that conjugates the group to one containing $\Gamma_{0}(n h)$.

The remaining sections are devoted to explaining notation and how the properties of the main theorem are obtained. We also explain (see Section 6) why some ideas of others are unsatisfactory as a contribution to our main theorem.

\section{The groups $\Gamma_{0}(n \| h)+\cdots$}

Let $N$ be a positive integer, and let $h$ be a positive integer such that $h^{2} \mid N$ and $h \mid 24$ with $N=n h$. Let $\Gamma_{0}(n \mid h)$ be the group of matrices of the form

$$
\left(\begin{array}{cc}
a & b / h \\
c n & d
\end{array}\right), \quad a d-b c n / h=1 .
$$


The group $\Gamma_{0}(n \mid h)$ is conjugate to $\Gamma_{0}(n / h)$ by $\left(\begin{array}{ll}h & 0 \\ 0 & 1\end{array}\right)$.

For each exact divisor $e$ of $N$ (we write $e \| N$ ), the Atkin-Lehner involution $W_{e}$ is the set of matrices $\left(\begin{array}{cc}a e & b \\ c N & d e\end{array}\right)$ with determinant $e$. Each $W_{e}$ is a single coset of $\Gamma_{0}(N)$. Moreover, the full normalizer of $\Gamma_{0}(N)$ in $\mathrm{PSL}_{2}(\mathbb{R})$ is obtained by adjoining to $\Gamma_{0}(n \mid h)$ its Atkin-Lehner involutions $w_{e}$ that are the conjugates by $\left(\begin{array}{ll}h & 0 \\ 0 & 1\end{array}\right)$ of the Atkin-Lehner involutions $W_{e}$ of $\Gamma_{0}(n / h)$.

The set of the exact divisors of $N, \operatorname{Ex}(N)$, is a group of exponent 2, where the group operation is given by $e * f=e f / \operatorname{gcd}(e, f)^{2}$. For each subgroup $\langle e, f, g, \ldots\rangle$ of $\operatorname{Ex}(n / h)$, we use the notation $\Gamma_{0}(n \mid h)+e, f, g, \ldots$ for the extension of $\Gamma_{0}(n \mid h)$ by its Atkin-Lehner involutions $w_{e}, w_{f}, w_{g}, \ldots$. This notation is abbreviated further by omitting " $h$ " when $h=1$, by writing $\Gamma_{0}(n \mid h)+$ when all of $\operatorname{Ex}(n / h)$ is involved, and by writing $\Gamma_{0}(n \mid h)$ - when no Atkin-Lehner involution is present.

The group $E(m)$ introduced earlier in this section has the form $\Gamma_{0}(n \mid h)+e$, $f, g, \ldots$ and the group $F(m)$ is a certain subgroup of $E(m)$ of index $h$, which we denote by $\Gamma_{0}(n \| h)+e, f, g, \ldots$. More explicitly, it was observed by Conway and Norton that $\Gamma_{0}(n \| h)+e, f, g, \ldots$ coincides with the kernel of the homomorphism $\lambda: \Gamma_{0}(n \mid h)+e, f, g, \ldots \rightarrow \mathbb{C}^{\times}$defined by:

(1) $\lambda=1$ for elements of $\Gamma_{0}(N)$,

(2) $\lambda=1$ for all Atkin-Lehner involutions $W_{E}$ of $\Gamma_{0}(N)$ for which every prime dividing $E$ also divides $n / h$,

(3) $\lambda=\exp (-2 \pi i / h)$ for cosets containing $\left(\begin{array}{cc}1 & 1 / h \\ 0 & 1\end{array}\right)$,

(4) $\lambda=\exp ( \pm 2 \pi i / h)$ for cosets containing $\left(\begin{array}{ll}1 & 0 \\ n & 1\end{array}\right)$, where the sign is + if $\left(\begin{array}{cc}0 & -1 \\ N & 0\end{array}\right)$ is present and - if not.

While the cosets in (3) and (4) generate $\Gamma_{0}(n \mid h)$, it is not clear that the definition of $\lambda$ is consistent. In other words, for arbitrary $n$ and $h$ as above, it is not clear that $\Gamma_{0}(n \| h)+e, f, g, \ldots$ exists.

\section{EXISTENCE AND STRUCTURE}

We now explain why the homomorphism $\lambda$ is well defined. As before, we are given a positive integer $N$ and $h$ such that $h^{2} \mid N$ and $h \mid 24$ and $n=N / h$. From the description of the normalizer of $\Gamma_{0}(N)$ given above, we see that $\Gamma_{0}(n \mid h)+e, f, g, \ldots$ normalizes $\Gamma_{0}(N)$, and since $\lambda=1$ on $\Gamma_{0}(N)$, we may ask whether $\lambda$ is well defined on the quotient $\Gamma_{0}(n \mid h)^{*}+e, f, g, \ldots:=\Gamma_{0}(n \mid h)+e, f, g, \ldots / \Gamma_{0}(N)$. In his thesis [10], Ferenbaugh investigated the structure of the quotient groups $\Gamma_{0}(n \mid h)^{*}$, which is as follows:

Let $x=\left(\begin{array}{ll}1 & 1 / h \\ 0 & 1\end{array}\right)$ and $y=\left(\begin{array}{ll}1 & 0 \\ n & 1\end{array}\right)$. Then we have

(1) If $h^{2} \mid n$ then $\Gamma_{0}(n \mid h)^{*} \cong C_{h} \times C_{h}$ where $x$ and $y$ are generators of the two copies of $C_{h}$.

(2) If $h^{2} \nmid n$ and $h$ is a prime power, then $\Gamma_{0}(n \mid h)^{*}$ is one of the groups:

- $\Gamma_{0}(2 \mid 2)^{*} \cong S_{3} \cong\left\langle x, y \mid x^{2}=y^{2}=(x y)^{3}=1\right\rangle$,

- $\Gamma_{0}(3 \mid 3)^{*} \cong A_{4} \cong\left\langle x, y \mid x^{3}=y^{3}=(x y)^{2}=1\right\rangle$,

- $\Gamma_{0}(4 \mid 4)^{*} \cong C_{2}^{2} \cdot S_{3} \cong\langle x, y, X, Y| x^{2}=X, y^{2}=Y, X^{2}=Y^{2}=1$, $\left.(x y)^{3}=1, x^{-1} Y x=y^{-1} Y y=X Y\right\rangle$,

- $\Gamma_{0}(8 \mid 4)^{*} \cong C_{2}^{2} \cdot C_{2}^{2} \cong\langle x, y, X, Y| x^{2}=X, y^{2}=Y, X^{2}=Y^{2}=1$, $\left.x y x^{-1} y^{-1}=X Y, x^{-1} Y x=Y, y^{-1} X y=X\right\rangle$,

- $\Gamma_{0}(8 \mid 8)^{*} \cong C_{4}^{2} \cdot S_{3} \cong\langle x, y, X, Y| x^{2}=X, y^{2}=Y, X^{4}=Y^{4}=1$, $\left.(x y)^{3}=1, x^{-1} Y x=X Y^{-1}, y^{-1} X y=X^{-1} Y\right\rangle$, 
- $\Gamma_{0}(16 \mid 8)^{*} \cong C_{4}^{2} \cdot C_{2}^{2} \cong\langle x, y, X, Y| x^{2}=X, y^{2}=Y, X^{4}=Y^{4}=1$, $\left.x y x^{-1} y^{-1}=X Y^{-1}, x^{-1} Y x=X^{2} Y, y^{-1} X y=X Y^{2}\right\rangle$,

- $\Gamma_{0}(32 \mid 8)^{*} \cong C_{4}^{2} \cdot C_{2}^{2} \cong\langle x, y, X, Y| x^{2}=X, y^{2}=Y, X^{4}=Y^{4}=1$, $\left.x y x^{-1} y^{-1}=X^{2} Y^{2}, x^{-1} Y x=Y, y^{-1} X y=X\right\rangle$.

Using the fact that $\Gamma_{0}(m n \mid h)^{*} \cong \Gamma_{0}(n \mid h)^{*} \times \Gamma_{0}(m \mid h)^{*}$ if $\operatorname{gcd}(m, n)=1$, any group $\Gamma_{0}(n \mid h)^{*}$ where $h$ is a prime power (that is, $h=2,3,4$ or 8 ) is isomorphic to one of these products.

(3) If $h$ has distinct prime divisors $(h=6,12$ or 24$)$ and $h=3 j$, then we have

$$
\begin{aligned}
\Gamma_{0}(n \mid h)^{*} & \cong \Gamma_{0}(3 n \mid j)^{*} \times \Gamma_{0}(j n \mid 3)^{*} \\
& \cong \Gamma_{0}(n / 3 \mid j)^{*} \times \Gamma_{0}(n / j \mid 3)^{*} .
\end{aligned}
$$

The structure of the quotient groups can be further detailed when we adjoin the Atkin-Lehner involutions. Indeed, the structure of $\Gamma_{0}(n \mid h)^{*}+e, f, g, \ldots$ can be derived from that of $\Gamma_{0}(n \mid h)^{*}$ as $\Gamma_{0}(n \mid h)^{*}+e, f, g, \ldots=\Gamma_{0}(n \mid h)^{*} \cdot C_{2}^{k}$, where $k$ is the number of generating Atkin-Lehner involutions. Furthermore,

(1) if $h$ is a prime power and with $x$ and $y$ as above, we have

$$
\begin{aligned}
& x^{w_{e}}= \begin{cases}x^{e} & \text { if } \operatorname{gcd}(e, h)=1, \\
y^{-N / e} & \text { if } h \mid e,\end{cases} \\
& y^{w_{e}}= \begin{cases}y^{e} & \text { if } \operatorname{gcd}(e, h)=1, \\
x^{-N / e} & \text { if } h \mid e .\end{cases}
\end{aligned}
$$

(Since $h$ is a prime power with $e \| N$ and $h \mid N$, it follows that either $\operatorname{gcd}(e, h)=1$ or $h \mid e$.

(2) If $h$ is not a prime power, then we express the group as a product of groups with a prime power $h$ as in $(*)$ or $(* *)$, and the action of any Atkin-Lehner involution is determined by its action on each component.

At this point we are able to check that the definition of $\lambda$ is compatible with the relations defining the groups and with the action of the Atkin-Lehner involutions. In other words, knowing the images of $x$ and $y$ and $w_{e}$, what are the various relations and actions conserved by the homomorphism $\lambda$ ?

It is not difficult to derive necessary and sufficient conditions for the consistency of the definition of $\lambda$. Some calculations for this are made in [10]. More precisely, for $N, h, n$ as above, the subgroup $\Gamma_{0}(n|| h)^{*}+e, f, g, \ldots$ of index $h$ in $\Gamma_{0}(n \mid h)^{*}+$ $e, f, g, \ldots$ exists if and only if the following conditions are satisfied:

(1) if $h=3$, then either $9 \mid n$ or $n / h \equiv \pm 1 \bmod h$, where the sign + is taken if $\tau \rightarrow 1 /(N \tau+1)$ is present and - otherwise;

(2) if $h=4$, then $8 \mid n$;

(3) if $h=8$, then $32 \mid n$;

(4) if $h$ is a prime power and $+e$ is present, then either $e \equiv 1 \bmod h$ or $n / e h \equiv \pm 1 \bmod h$. The sign + is taken if $\tau \rightarrow 1 /(N \tau+1)$ is present and - otherwise;

(5) if $h=6,12,24$, then both subgroups in the direct product $(*)$ or $(* *)$ satisfy all the above four conditions.

\section{THE GENUS ZERO PROPERTY}

The above existence properties provide infinitely many groups of the form $\Gamma_{0}(n \| h)+e, f, g, \ldots$ In this section we determine when these groups have genus zero. 
TABLE 1. The 123 genus zero groups of the form $\Gamma_{0}(N)+e, f, g, \ldots$

\begin{tabular}{|c|c|c|}
\hline $1+$ & $17+$ & $36+36$ \\
\hline $2-$ & $18-$ & $36+$ \\
\hline $2+$ & $18+2$ & $38+$ \\
\hline $3-$ & $18+9$ & $39+39$ \\
\hline $3+$ & $18+18$ & $39+$ \\
\hline $4-$ & $18+$ & $41+$ \\
\hline $4+$ & $19+$ & $42+3,14,42$ \\
\hline $5-$ & $20+4$ & $42+6,14,21$ \\
\hline $5+$ & $20+20$ & $42+$ \\
\hline $6-$ & $20+$ & $44+$ \\
\hline $6+2$ & $21+3$ & $45+$ \\
\hline $6+3$ & $21+21$ & $46+23$ \\
\hline $6+6$ & $21+$ & $46+$ \\
\hline $6+$ & $22+11$ & $47+$ \\
\hline $7-$ & $22+$ & $49+$ \\
\hline $7+$ & $23+$ & $50+50$ \\
\hline $8-$ & $24+8$ & $50+$ \\
\hline $8+$ & $24+24$ & $51+$ \\
\hline $9-$ & $24+$ & $54+$ \\
\hline $9+$ & $25-$ & $55+$ \\
\hline $10-$ & $25+$ & $56+$ \\
\hline $10+2$ & $26+26$ & $59+$ \\
\hline $10+5$ & $26+$ & $60+4,15,60$ \\
\hline $10+10$ & $27+$ & $60+12,15,20$ \\
\hline $10+$ & $28+7$ & $60+$ \\
\hline $11+$ & $28+$ & $62+$ \\
\hline $12-$ & $29+$ & $66+6,11,66$ \\
\hline $12+3$ & $30+15$ & $66+$ \\
\hline $12+4$ & $30+2,15,30$ & $69+$ \\
\hline $12+12$ & $30+3,5,15$ & $70+10,14,35$ \\
\hline $12+$ & $30+5,6,30$ & $70+$ \\
\hline $13-$ & $30+6,10,15$ & $71+$ \\
\hline $13+$ & $30+$ & $78+6,26,39$ \\
\hline $14+7$ & $31+$ & $78+$ \\
\hline $14+14$ & $32+$ & $87+$ \\
\hline $14+$ & $33+11$ & $92+$ \\
\hline $15+5$ & $33+$ & $94+$ \\
\hline $15+15$ & $34+$ & $95+$ \\
\hline $15+$ & $35+35$ & $105+$ \\
\hline $16-$ & $35+$ & $110+$ \\
\hline $16+$ & $36+4$ & $119+$ \\
\hline
\end{tabular}

A necessary condition for a group $\Gamma_{0}(n \| h)+e, f, g, \ldots$ to be of genus zero is that the supergroup $\Gamma_{0}(n \mid h)+e, f, g, \ldots$ is of genus zero. Moreover, $\Gamma_{0}(n \mid h)+$ $e, f, g, \ldots$ is conjugate to $\Gamma_{0}(n / h)+e, f, g, \ldots$ Thus, we need to list the groups $\Gamma_{0}(n / h)+e, f, g, \ldots$ of genus zero, which in fact correspond to $h=1$, and then, when $h$ is introduced, find out which subgroups $\Gamma_{0}(n \| h)+e, f, g, \ldots$ are of genus zero. Fricke [13] gives a partial list of $\Gamma_{0}(N)+N$ which are of genus zero, and Ogg [19] showed that Fricke's list is complete when $N$ is prime, by connecting them to supersingular elliptic curves. It turns out that those primes are exactly the prime 
divisors of the order of the Monster $\mathbb{M}$; this connection is yet to be explained. In [18], Kluit gives the full list of the groups $\Gamma_{0}(N)+e, f, g, \ldots$ that are of genus zero. In fact, by connecting the genus of these groups to dimensions of spaces of modular forms, he showed that these groups are the same ones that appear in Table 5 of 2]. There are 123 such groups which we list in Table 1 . In this table we use the notation $N+e, f, g, \ldots$ for the group $\Gamma_{0}(N)+e, f, g, \ldots$, together with the notation of Section 3.

As for the groups of the form $\Gamma_{0}(N|| h)+e, f, g, \ldots$, the genus can be determined from their fundamental regions using the Riemann-Hurwitz formula. Since most of the groups are not subgroups of the modular group, the calculations of the genus, which cannot be produced here because of their length, are carried out by finding the elliptic fixed points and the cone points in the orbifolds attached to the fundamental regions. The Euler characteristic of the orbifold determines the genus of the group. See [6] for more details on orbifold techniques. We note that the genus zero groups of the form $\Gamma_{0}(n \| h)+e, f, g, \ldots$ are exactly those of the same form in [12], thus confirming a part of Norton's conjecture on replicable functions.

\section{THE EXPONENT TWO PROPERTY}

The number of the groups $\Gamma_{0}(n \| h)+e, f, g, \ldots$ of genus zero is 213 , which includes the 171 groups of Moonshine. Here we find properties that distinguish these 171 Moonshine groups from the others. A first remark in this regard is that when $h=1$, the quotient of each group by the underlying $\Gamma_{0}(N)$ is a group of exponent 2 since we adjoin only Atkin-Lehner involutions. Also in our list of genus zero groups we have both $\Gamma_{0}(2 \| 2)$ and $\Gamma_{0}(3 \| 3)$; however, the former is not in the Moonshine list, while the latter is. From the index formula we see that $\Gamma_{0}(3 \| 3) / \Gamma_{0}(9)$ has order 4 , and in fact it is $C_{2} \times C_{2}$, while $\Gamma_{0}(2 \| 2) / \Gamma_{0}(4)$ has order 3 . This leads us to ask whether the right property to look for is that the quotient of $\Gamma_{0}(n \| h)+e, f, g, \ldots / \Gamma_{0}(n h)$ is an elementary abelian 2 -group, that is, a group of exponent 2. In fact, simple criteria can be developed to remove at once many groups whose quotient by the underlying $\Gamma_{0}(N)$ is not of exponent 2 . Recall that $N=n h$ and $h \mid n$. Let $x=\left(\begin{array}{ll}1 & 1 / h \\ 0 & 1\end{array}\right)$ and $y=\left(\begin{array}{ll}1 & 0 \\ n & 1\end{array}\right)$. As we saw earlier, the cosets containing these two elements generate $\Gamma_{0}(n \mid h)$. From the definition of the homomorphism $\lambda$ in Section 3, we see that $x y \in \Gamma_{0}(n \mid h)$ if the Fricke element $\left(\begin{array}{lr}0 & -1 \\ N & 0\end{array}\right)$ is present and $x y^{-1} \in \Gamma_{0}(n \mid h)$ if not. It follows that

$$
\left(x y^{ \pm}\right)^{2}=\left(\begin{array}{cc}
\left(1 \pm \frac{n}{h}\right)^{2} \pm \frac{n}{h} & \frac{1}{h}\left(2 \pm \frac{n}{h}\right) \\
\pm n\left(2 \pm \frac{n}{h}\right) & \pm \frac{n}{h}+1
\end{array}\right),
$$

and therefore, a necessary and sufficient condition for $\left(x y^{ \pm}\right)^{2}$ to be in $\Gamma_{0}(N)$ is that $h \mid(2 \pm n / h)$. Furthermore, this should be the case if the quotient $\Gamma_{0}(n \| h)^{*}=$ $\Gamma_{0}(n \| h) / \Gamma_{0}(N)$ is a group of exponent 2. Notice that we do not need to deal with the Atkin-Lehner involutions since they do not affect this property. The condition $h \mid(2 \pm n / h)$ allows us to determine many groups for which the exponent 2 property is not satisfied. We can improve this criterion by looking at $\left(x^{k} y^{ \pm k}\right)^{2}$ instead. However, the remaining cases are dealt with by investigating whether the quotients $\Gamma_{0}(n \mid h) / \Gamma_{0}(N)$ described in Section 4 have normal subgroups of index $h$ and exponent 2 . 


\section{The GHosts}

We are now left with 174 genus zero groups of the form $\Gamma_{0}(n \| h)+e, f, g, \ldots$ of exponent 2. These are the 171 of Monstrous Moonshine and the three groups $\Gamma_{0}(25)-, \Gamma_{0}(49)+$ and $\Gamma_{0}(50)+50$ known as the ghosts. Conway and Norton noted that these three ghosts are distinguished by not having product formulas; it was also suggested by Akbas and Singerman 1 that the reason for these ghosts not being in Moonshine is that the normalizers of the underlying $\Gamma_{0}(N)$ in $\mathrm{PSL}_{2}(\mathbb{R})$ are not transitive on cusps. While true, this property holds also for $\Gamma_{0}(25)+$ and $\Gamma_{0}(50)+$, which are in Moonshine. Even the question whether the normalizers of the groups themselves, rather than the underlying $\Gamma_{0}(N)$, are transitive on cusps adds more problems with two other groups of different levels, namely $\Gamma_{0}(12)+12$ and $\Gamma_{0}(27)+$, which have normalizers not transitive on the cusps, and yet they are in the Moonshine list. Hence, the transitivity on the cusps is not an explanation for the ghosts not being in the Moonshine list.

Nonetheless, the properties of the cusps seem to be the key to dealing with the ghosts. The Moonshine groups all share the common property of having cusp width 1 at $\infty$, as exhibited by the $q$-expansion of their principal moduli about $\infty$; and the natural width at $\infty$ for the Moonshine groups seems to be 1 .

What is the natural width for the other cusps?

Each cusp for one of the groups in question can be conjugated to $\infty$ by an element of $\mathrm{PSL}_{2}(\mathbb{R})$. In order for the group to have a principal modulus with a cusp at $\infty$ after conjugation, it should contain a certain $\Gamma_{0}\left(N^{\prime}\right)$ and have translations generated by $\tau \mapsto \tau+1$. Examining a few examples reveals that it is always possible to find a conjugation where $N^{\prime}$ is equal to $N$, the level of the underlying $\Gamma_{0}(N)$ of the original group. Surprisingly, the only groups that do not satisfy this property are the ghosts. Indeed, those that have a normalizer transitive on the cusps satisfy this property automatically, and the few other groups in the Moonshine list whose normalizers are not transitive on cusps can be shown to have the same property. Thus, a cusp will have the natural width if it can be conjugated to $\infty$ by an element of $\mathrm{PSL}_{2}(\mathbb{R})$ for which the conjugated group still contains $\Gamma_{0}(N)$. Of course, the fact that the normalizers are transitive on the cusps implies that this property is true for any cusp.

\section{REFERENCES}

[1] M. Akbas and D. Singerman, The signature of the normalizer of $\Gamma_{0}(N)$. Groups, Combinatorics and Geometry (Durham, 1990), 77-86, London Math. Soc. Lecture Note Ser., 165, Cambridge University Press, Cambridge, 1992. MR 94a:20081

[2] B. J. Birch and W. Kuyk, eds., Modular functions of one variable IV, Proc. Internat. Summer School (Antwerp, 1972), Lecture Notes in Mathematics, no. 476, Springer-Verlag, New York, 1975. MR 51:12708

[3] R. Borcherds, Monstrous Moonshine and Monstrous Lie superalgebras, Invent. Math. 109 (1992), 405-444. MR 94f:11030

[4] R. Borcherds, Automorphic forms on $O_{s+2,2}(R)$ and infinite products, Invent. Math. 120 (1995), 161-213. MR 96j:11067

[5] J. H. Conway, Understanding groups like $\Gamma_{0}(N)$. Groups, Difference Sets, and the Monster (Columbus, OH, 1993), 327-343, Ohio State Univ. Math. Res. Inst. Publ., 4, de Gruyter, Berlin, 1996. MR 98b:11041

[6] J. H. Conway, The orbifold notation for surface groups. Groups, Combinatorics and Geometry (Durham, 1990), 438-447, London Math. Soc. Lecture Note Ser., 165, Cambridge University Press, Cambridge, 1992. MR 94a:57025 
[7] J. H. Conway and S. P. Norton, Monstrous Moonshine, Bull. London Math. Soc. 11 (1979), 308-339. MR 81j:20028

[8] C. Dong, H. Li, and G. Mason, Modular-invariance of trace functions in orbifold theory and generalised Moonshine, Commun. Math. Phys. 214 (2000), 1-56. MR 2001k:17043

[9] C. Dong and G. Mason, An orbifold theory of genus zero associated to the sporadic group $M_{24}$, Comm. Math. Phys. 164 (1994), no. 1, 87-104. MR 96a:11041

[10] C. Ferenbaugh, On the modular functions involved in "Monstrous Moonshine", Ph.D. dissertation, Princeton University, 1992.

[11] I. Frenkel, J. Lepowsky, and A. Meurman, Vertex operator algebras and the Monster, Pure and Applied Mathematics, vol. 134, Academic Press, Inc., Boston, MA, 1988. MR 90h:17026

[12] D. Ford, J. McKay and S. Norton, More on replicable functions, Comm. Algebra 22 (1994), 5175-5193. MR 95i:11036

[13] R. Fricke, Die elliptische Funktionen und ihre Anwendungen, 2-ter Teil (Teubner, Leipzig, 1892).

[14] R. Griess, The friendly giant, Invent. Math. 69 (1982), 1-102. MR 84m:20024

[15] J. Harvey and G. Moore, Algebras, BPS states, and strings, Nucl. Phys. B463 (1996) 315-368. MR 97h:81163

[16] Y.-Z. Huang, Two-dimensional conformal geometry and vertex operator algebras, Progress in Mathematics, 148, Birkhäuser Boston, Inc., Boston, MA, 1997. MR 98i:17037

[17] R. Ivanov and M. Tuite, Rational generalised Moonshine from Abelian orbifoldings of the Moonshine module, Nucl. Phys. B635 (2002), 435-472. MR 2003g:11040

[18] P. G. Kluit, On the normalizer of $\Gamma_{0}(N)$, in Modular Functions of One Variable, V (Bonn, 1976), 239-246, Lecture Notes in Mathematics, no. 601, Springer-Verlag, New York, 1977. MR 58:513

[19] A. P. Ogg, Automorphismes des courbes modulaires, Séminaire Delange-Pisot, Poitou, (7), 1974.

[20] M. Tuite, Monstrous Moonshine from orbifolds, Comm. Math. Phys. 146 (1992), no. 2, 277309. MR 93f: 11036

[21] M. Tuite, On the relationship between Monstrous Moonshine and the uniqueness of the Moonshine Module, Commun. Math. Phys. 166 (1995), 495-532. MR 96b:17027

Department of Mathematics, Fine Hall, Princeton University, Washington Road, Princeton, New Jersey 08544-1000

E-mail address: conway@math.princeton.edu

Department of Mathematics and CiCMA, Concordia University, 1455 de Maisonneuve Blvd. West, Montreal, Quebec H3G 1M8, Canada

E-mail address: mckay@cs.concordia.ca

Department of Mathematics and Statistics, University of Ottawa, Ottawa, Ontario K1N 6N5, CANADA

E-mail address: sebbar@mathstat.uottawa.ca 